\title{
Deployment and Reallocation in Mobile Survivability-Heterogeneous Wireless Sensor Networks for Barrier Coverage
}

\author{
Jie Tian, Xiaoyuan Liang, and Guiling Wang \\ Department of Computer Science, New Jersey Institute of Technology \\ Email: $\{$ jt66,xl367,gwang $\} @$ njit.edu
}

\begin{abstract}
Barrier coverage is a widely adopted coverage model for intruder surveillance application in wireless sensor networks. However, when sensor nodes are deployed outdoors, they are subject to environmental detriments and will be failed while operating in the rain. Thus, one barrier is not robust to provide barrier coverage under both sunny and rainy weather. In this paper, we study the barrier coverage problem in a mobile survivability-heterogeneous wireless sensor network, which is composed of sensor nodes with environmental survivabilities to make them robust to environmental conditions and with motion capabilities to repair the barrier when sensors are dead. Our goal is to keep field to be monitored continuously under both sunny and rainy weather and to prolong the network lifetime as much as possible. We propose a novel greedy barrier construction algorithm to solve the problem. The algorithm adopts weather forecast to direct the barrier construction under sunny and rainy weather, and the energy consumption of construction is minimized. Simulation results show that our algorithm efficiently solves the problem and outperforms other alternatives.

Keywords: Survivability-Heterogeneous Wireless Sensor Networks, Mobile Sensor Networks, Barrier Coverage
\end{abstract}

\section{INTRODUCTION}

Wireless Sensor Networks have been widely employed in many long-term surveillance applications such as field surveillance, critical infrastructure protection and country border control. Intruder detection as a very important long-term field surveillance application is extensively studied in the community [1]-[3]. In the application, sensors are deployed in the area to detect intruders. The intruder's objective is to traverse the monitored area. For example, in a battlefield, an enemy may try to traverse a protected area to conduct some malicious tasks, the successful detection of which before he passes through is important.

Among intruder surveillance applications, barrier coverage is a widely known coverage model to detect intruders. A barrier is a line of sensors across the entire field of interest. The sensing ranges of two neighbor sensors in the barrier are overlapped and thus the intruder are guaranteed to be detected. In the applications, the sensors are always deployed outdoors to achieve barrier coverage. However, as a tiny electronic device, an on-duty sensor node is vulnerable to many environmental attributes or detriments, such as rain or snow [4]. The sensor nodes may become unreliable and even dead under the rain, and thus the barrier coverage cannot be guaranteed. The environmental problem has been frequently cited as a vital reason to destroy the barrier coverage, but very few research efforts have been dedicated to addressing the problem directly or fundamentally. Another problem in barrier construction outdoors is that it is almost impossible to construct an optimal barrier with sensors side by side along a straight line across the region, when sensors cannot move and are randomly distributed in the network.

To increase the survivability of a sensor node outdoor, one way is to equip it with additional protection to make it for example water-proof [5]. However, to make all sensor nodes water-proof is not cost-effective. It may greatly increase the cost of sensor networks, making them less applicable [6]. Also, many environmental conditions that can do harm to sensor nodes only happen occasionally in an area. For example, in a given area, there is a low probability that it continuously rains for a long time. Hence, an effective solution is to construct a sensor network composed of both regular sensor nodes lacking of protection and nodes with additional environmentally robust features [7]. We call the latter environmentally robust nodes, or robust nodes for short. The term environmental survivability characterizes such heterogeneity, which is fundamentally different from the widely studied heterogeneity in terms of computational capacity, energy, communication range, and sensing range [8]-[11].

To efficiently construct barriers under a random sensor distribution, robust sensors with controlled mobility are employed in the network to guarantee the coverage. Since regular sensors can be damaged during the rain, it is not reasonable to add motor bases to make them move, which is costly. For robust sensors, adding motor bases can fully explore the better use of the robust feature when needed. After the initial deployment, the mobile robust sensors can move to certain locations and connect with other un-utilized sensors to form barriers under both sunny and rainy weather. This paper proposes a new kind of wireless sensor network, a mobile survivability-heterogeneous wireless sensor network composed of both static regular sensors without protection and mobile robust sensors with protection. It is important to exploit the moving strategy of mobile robust sensors to achieve barrier coverage under different weather conditions.

To tackle the problem, we leverage the mix deployment of 
static regular sensors and mobile robust sensors to construct barriers under different weather conditions. Our objective is to construct one barrier with minimum sensing overlaps to detect intruders as long as possible under both sunny and rainy weather. Note that static regular sensors cannot work under the rain; otherwise they will be damaged permanently and cannot work any more though they still have energy left. Mobile robust sensors need to conserve their energy consumption such that they still have enough energy to move and thus to form a working barrier when there comes rain. Since the energy consumption of movement in mobile robust sensors is much higher than energy consumption of sensing and communication, the aim of this paper is to minimize the total energy consumption of movement of robust sensors to construct barriers under different weather conditions such that the network lifetime is prolonged as much as possible.

Because of the additional dimension of complexity in barrier construction incurred by weather, previous works [1], [2], [12]-[14] in studying barrier construction to achieve guaranteed barrier coverage cannot be directly employed here. If all static regular sensors are used to construct the barrier under the sunny weather, a large amount of mobile robust sensors need to be moved to form a new barrier when rain comes and thus a lot of energy is consumed. For example, in [13], a lot of mobile sensors need to move to the predefined line under the rainy weather to form a new barrier. If all mobile robust sensors are used to construct the barrier at the beginning, they will work for a long time and will not have enough energy to work during rainy weather. Thus, our idea is to use both static regular sensors and mobile robust sensors to construct a barrier with the least energy consumption under sunny weather and then to reallocate mobile robust sensors to construct a new barrier with the least energy consumption in robust sensors' movement under rainy weather.

In the paper, we propose a greedy barrier construction algorithm to solve the problem. Given a long-belt shaped area, a barrier is first constructed in a centralized manner without moving any robust sensors. When rain comes, a new barrier is constructed locally to save energy consumption of communication. The long-belt area is divided into several grids based on the positions of mobile robust sensors in the barrier. In each grid, a coordinator is selected to direct the movement of mobile robust sensors to construct a new barrier. If there are not enough mobile robust sensors to reconstruct the barrier, the grid merges itself with one of its neighbor grids to form a bigger grid to make the reconstruction. To increase the network lifetime, minimizing energy consumption of moving robust sensors in each grid is considered by each coordinator to make the decision. fold:

To summarize, our contribution in this paper is three-

- We for the first time propose a new mobile survivabilityheterogeneous sensor networks composed of both static sensors nodes without protection and mobile sensors nodes with environmentally robust features.
- We for the first time study the barrier coverage problem in such heterogeneous WSN considering different weather conditions.

- A greedy algorithm is proposed to achieve efficient and effective barrier coverage under both rainy and sunny weather.

The remainder of the paper is organized as follows. Related work is discussed in Section II. We present the assumptions and models in Section III. Section IV describes the greedy algorithm. Section V reports simulation results. Finally, we conclude the paper in Section VI.

\section{RELATED WORK}

Barrier coverage for intruder detection has been studied extensively. Theoretical foundations of designing barriers using wireless sensors in WSNs are developed in [15]. According to [15], the barrier coverage problem is very difficult to solve in a decentralized way due to its globalized nature. Chen et al. address this challenge by introducing the concept of local barrier coverage in [16]. In [17], Liu et al. propose a distributed algorithm to construct multiple disjoint barriers for strong barrier coverage when sensors are distributed following a Poisson distribution. In [18], Saipulla et al. study the barrier coverage problem when sensors are deployed along a line. To further utilize the barrier concepts in wireless sensor networks, Kumar et al. develop solutions for the case when sensors are deployed to form an impenetrable barrier for detecting movements in [19]. In [20], Chen et al. introduce a concept of local barrier coverage and propose a localized barrier coverage protocol. The concept of one-way barrier coverage is introduced in [21], which requires the network reports illegal intruders while ignores legal intruders. Scheduling for barrier coverage is studied in [19], [22]. In [23], Yang et al. split sensor deployment into multiple rounds to reduce the number of sensors needed to provide guaranteed barrier coverage.

There are also existing works on intruder detection by using a mix deployment of mobile and static sensors or only mobile sensors. Paul et al. present a cluster based scheduling algorithm considering mobility of nodes in [24]. In [25], mobile sensors are used to provide k-barrier coverage against moving intruders. Saipulla et al. study how to efficiently improve barrier coverage using mobile sensors with limited mobility in [13]. Tian et al. propose a dynamic intruder detection scheme using mobile and static sensors to detect smart intruders [26]. He et al. use mobile sensors to achieve barrier coverage in sensor scarcity case by dynamic sensor patrolling in [27]. Cheng et al. use only mobile sensors to construct barriers based on sensor's density in [14].

However, none of the existing work focuses on providing barrier coverage considers environmental attributes, such as rain or snow, which is an important factor to construct barrier and then to determine the network lifetime. Without such consideration, the previous schemes cannot be directly applied to solve the problem in this paper. 


\section{AsSUMPTIONS AND MODELS}

\section{A. Assumptions}

In the paper, we make following assumptions:

- There are two types of sensors in the network, static regular sensor and mobile robust sensor. A mobile robust sensor is reliable and can work under both sunny and rainy weather. It can also move without any movement constraints under different weather conditions. A static regular sensor can only work under sunny weather and can be damaged with a probability during the rain. After the static regular sensor dries out, it can function normally again [28].

- Every sensor knows its location, which can be obtained by GPS or localization methods [29]-[31].

- An isotropic sensing model is adopted [21], [32]. The sensing area of each sensor node is a circle with the same radius, $R S$. If the occurrence of the intruder is within the sensing range of a sensor node, then the intruder is assumed to be detected, otherwise not. The transmission range of a sensor is always more than twice the sensing range [17]. Such model is also well known as $0 / 1$ model $^{1}$.

- The energy consumption of starting motor and moving is considered. The energy model in [33] is adopted for mobile robust sensors:

$$
P=P_{s}+P_{e} \times s,
$$

where $P$ is the total power consumption of movement, $P_{s}$ is the energy consumption to start the motor, $P_{e}$ is the energy consumption per unit of moving, and $s$ is the total moving distance.

- Current weather forecast techniques are mature and can provide accurate weather forecast information of a given area [4], [34]. Specifically, weather forecast provides the information that whether or not there is going to be rainy in the next several hours. So future weather information is assumed to be available in the network and it is $100 \%$ accurate [7].

\section{B. Barrier Coverage Model}

Without loss of generality, we consider a long-belt shaped field to be monitored. The objective of intruders is to cross the field vertically from one side following a crossing path. A crossing path is a path that connects one side of the region to the opposite side, which is first defined in [17]. We do not impose any constraints on intruders in terms of moving direction, moving speed, departing time, and other factors.

A barrier is a chain of overlapping sensors across the entire region. The barrier can intersect any crossing paths and thus is guaranteed to detect any crossing intruders. The definition of barrier coverage in [17] is introduced as follows:

DEFINITION 3.1: A path is said to be 1-covered if it intercepts at least one distinct sensor.

\footnotetext{
${ }^{1}$ For other models like probability sensing models, they still work effectively. One possible solution is to set a threshold as the positive detection probability in the probability models, which can be used to determine whether or not an intruder is detected.
}

DEFINITION 3.2: A sensors network is said to be strongly 1-barrier covered if:

$$
P(\text { any crossing path is } 1 \text {-covered })=1 \text { w.h.p. }
$$

In the paper, we study constructing one strong barrier in the network. The challenges lying in the construction are: guarantee a barrier working under both sunny and rainy weather and prolong network lifetime as much as possible.

\section{Algorithm}

In this section, a greedy algorithm is proposed to construct a barrier in the proposed heterogenous sensor networks by adopting weather forecast. We first present the overview of the algorithm. Then the detailed algorithm is discussed.

\section{A. Overview}

In the real world, weather conditions change all the time. A barrier containing only regular sensors has a high probability to function unsuccessfully under consecutive sunny and rainy periods, since regular sensors may be destroyed while running under rainy weather. An intuitive way is to construct two barriers and let them work under sunny and rainy weather rotationally. One of them is only composed of regular sensors and works under the sunny weather. Another is only composed of robust sensors and works under the rainy weather. However, it is possible that the first barrier cannot be constructed when the density of sensors is not high enough, and a large number of robust sensors need to be moved to construct the second barrier under the rainy weather without considering the distribution. A large amount of energy will be consumed on mobile sensors' movement and thus the network lifetime will decrease. Our idea is that we use both regular sensors and robust sensors to construct a barrier working under sunny weather. The mobile robust sensors only move if they have to, such as filling coverage holes. When rain comes, a new barrier is locally built on the previous barrier by reallocating some mobile robust sensors. Since there are already robust sensors in the barrier, only a small number of robust sensors need to be moved to construct the new barrier and the energy consumption of movement in mobile robust sensors is largely reduced. Furthermore, since reallocation is performed locally, the communication energy consumption is also reduced. When the sunny weather comes again, both regular sensors and robust sensors will be used to reconstruct a new barrier.

A greedy algorithm is proposed to achieve the idea. It is composed of three algorithms: Default Barrier Construction algorithm, Mobile Sensor Reallocation algorithm and Barrier Reconstruction algorithm. In Default Barrier Construction algorithm, a barrier is first constructed with both regular sensors and robust sensors based on network topology. If there is any coverage hole in the network because of initial deployment or new generation from sensors' failure, the mobile robust sensors are used to bridge the sensors on two sides of the hole. The constructed default barrier works only under the sunny weather. When rain comes, Mobile Sensor Reallocation algorithm is executed. Only mobile robust sensors are used to construct a new barrier, since regular sensors don't work 
during the rain. The reallocation includes two phases: (1) grid division and merging, and (2) virtual movement evaluation. Based on the calculation results of reallocation algorithm, a number of mobile robust sensors move to calculated positions to construct the barrier. When sunny weather comes again, regular sensors come back to work. Barrier Reconstruction algorithm is executed to rebuild a barrier based on previous two barriers. The details of the three algorithms are presented in the following sections.

\section{B. Default Barrier Construction Algorithm}

The main goal of this algorithm is to construct a default barrier with least movement of mobile robust sensors. We consider all robust sensors to be static sensors. There are several existing works on using only static sensors to construct a barrier [15], [17], [22]. In our algorithm, the centralized algorithm in [17] is adopted to construct the default barrier. One example of the default barrier is shown in Fig. 1.

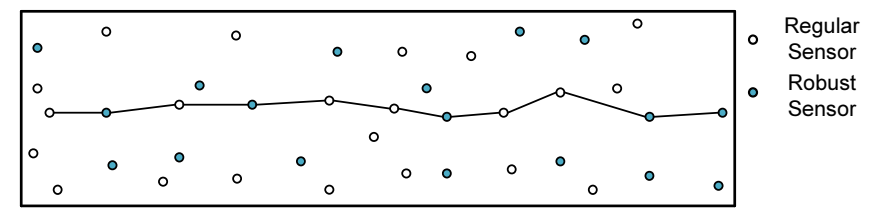

Fig. 1. Default Barrier

When sensors are deployed dense enough, by leveraging the centralized algorithm in [17], the default barrier can be constructed successfully. But when the distribution of sensors is not dense enough, there is a probability that the barrier cannot be constructed, and coverage holes exist in the network as shown in Fig. 2(a). In such case, we use mobile robust sensors to fill the holes and bridge sensors located in different parts of the field. The modified algorithm is still performed in a centralized way as its original version.

The coverage holes separate the network into several unconnected components. Assume the algorithm starts the construction from left component. It constructs the default barrier in the left component and stops at a node on the left side of a coverage hole. The sensor node, which the construction ends at, is called a stopping node. Then a straight path between the stopping node and left most sensor in the right component is determined. Several positions on the path are calculated, the distance between any two neighbors of which is $2 \times R S$ considering minimizing sensing overlaps. For each position, the closest robust sensor is moved to it. The movement cannot destroy the already constructed default barrier. After the movement, the barrier reaches the left most sensor in the right component. The barrier construction continues.

One example is shown in Fig. 2. In Fig. 2(a), Default Barrier Construct algorithm stops at node $s_{1}$, since there is a coverage hole on its right side. Node $s_{1}$ is a stopping node. Then the algorithm builds a straight path between node $s_{1}$ and node $s_{4}$, since node $s_{4}$ is the left most sensor on the right side of coverage hole as shown in Fig. 2(b). Two positions are calculated on the path also as shown in Fig. 2(b). The robust

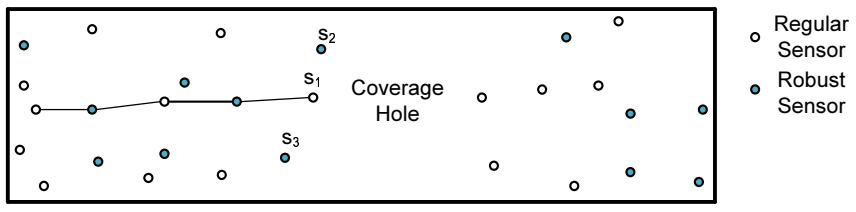

(a) Coverage Hole Detection

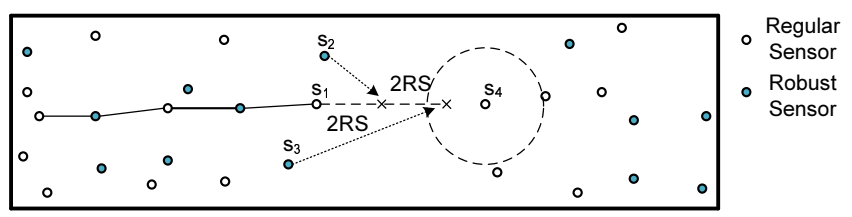

(b) Position Calculation

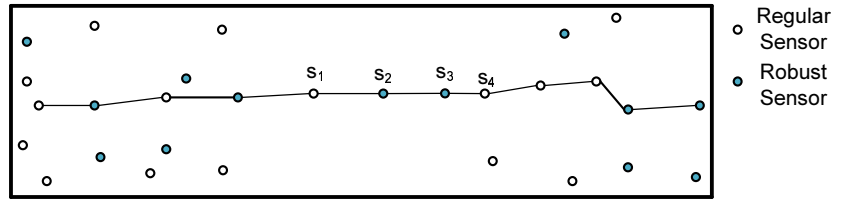

(c) Mobile Robust Sensor Movement

Fig. 2. Repairing Coverage Hole

sensor nodes $s_{2}$ and $s_{3}$ are moved to the positions, since they are closest to the positions. After the movement, node $s_{3}$ reaches node $s_{4}$ and then the algorithm continues to construct the default barrier as shown in Fig. 2(c).

In the construction, the mobile robust sensors only move when there are coverage holes in the network. After the default barrier is constructed, it is responsible for monitoring the field under sunny weather.

\section{Mobile Sensor Reallocation Algorithm}

When rain comes, the default barrier doesn't function any longer, since regular sensors don't work under the rain. A new barrier needs to be constructed using only mobile robust sensors. Mobile Sensor Reallocation algorithm is responsible for constructing a new barrier locally. There are two steps in the algorithm: (1) grid division and merging, and (2) virtual movement evaluation.

1) Grid Division and Merging: The long-belt shaped area is first horizontally divided into grids. When dividing the grids, each robust sensor in the barrier checks the types of its barrier neighbor sensors. There are four different cases in the barrier as shown in Fig. 3: (1) left neighbor is a regular sensor and right neighbor is a robust sensor; (2) left neighbor is a robust sensor and right neighbor is a regular sensor; (3) both left and right neighbors are regular sensors; and (4) both left and right neighbors are robust sensors. In cases (1), (2) and (3), the current robust sensor is defined as an edge node. The vertical line across it is the shared edge between two neighbor grids. In case (4), the current robust sensor is not an edge node. For example, in Fig. 3(a), regular sensor $s_{1}$ is the left neighbor of robust sensor $s_{2}$, and robust sensor $s_{3}$ is the right neighbor of robust sensor $s_{2}$. The vertical line crossing $s_{2}$ is the right edge of grid $a$ and left edge of grid $b$, and $s_{2}$ is the edge node of both grids. Similarly, $s_{2}$ is the edge node of both grid 


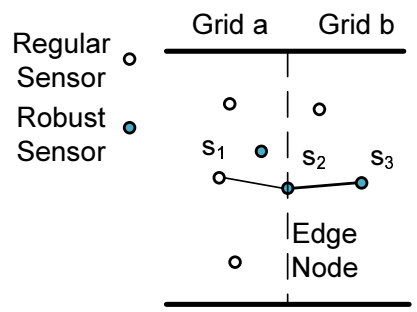

(a) Case 1

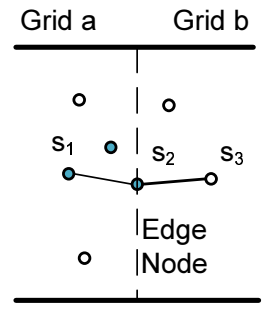

(b) Case 2

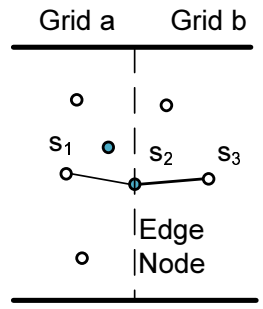

(c) Case 3

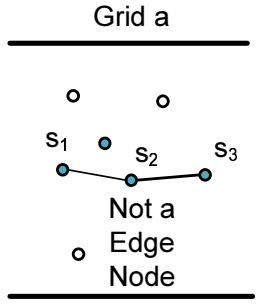

(d) Case 4

Fig. 3. Edge Node Calculation

$a$ and $b$ in Fig. 3(b) and (c). In Fig. 3(d), since both $s_{1}$ and $s_{3}$ are robust sensors, $s_{2}$ is not an edge node. It is obvious that a robust sensor is an edge node only if it has at least one regular sensor neighbor in the barrier. After the edge nodes are determined, the network is divided into several grids. One example of grid division is shown in Fig. 4.

After the division, there are two kinds of grids: one contains a segment of barrier only composed of regular sensors and another contains a segment of barrier only composed of robust sensors. Mobile robust sensors in the second kind of grid are scheduled to be working under the coming rainy periods. In the first kind of grid, a new segment of barrier needs to be constructed. To direct the movement of robust sensors, a coordinator is selected in each grid. The functions of the coordinator include: 1 ) merging grids by communicating with other coordinators, 2) choosing mobile robust sensors to construct the segment of barrier, and 3) directing mobile robust sensors to move to certain positions.

The coordinator is selected from one of two edge nodes of a grid. When a robust sensor is an edge node of a grid, it must also be the edge node of the neighbor grid. As shown in Fig. 4, the robust sensor $s_{1}$ is the right edge node of grid 2 and also the left edge node of grid 3. Similarly, robust sensor $s_{2}$ is the right edge node of grid 3 and also the left edge node of grid 4 . Clearly, each edge node can obtain two neighbor grids' information. To select a coordinator in one grid, both edge nodes add the difference between the number of available mobile robust sensors and the number of required mobile robust sensors to reconstruct the segment of barrier in the current grid and neighbor grid together. The number of required mobile robust sensors is the number of regular sensors in the segment of barrier. The edge node with the highest summation value is selected as the coordinator, since it can direct more mobile robust sensors than another edge node to reconstruct the barrier in current grid. If the summation values are the same for both edge nodes, a random edge node is selected as the coordinator. Let $m$ denote the number of available mobile robust sensors, $n$ denote the number of required mobile robust sensors and $\Delta n$ denote the difference in one grid, where $\Delta n=m-n$. As the example shown in Fig. 5(a), $\Delta n_{a}=-1$ in grid $a, \Delta n_{b}=1$ in grid $b$, and $\Delta n_{c}=2$ in grid $c$. To select the coordinator in grid $a$, sensor $s_{1}$ calculates $\Delta n_{a}+\Delta n_{b}=0$, and sensor $s_{2}$ calculates $\Delta n_{a}+\Delta n_{c}=1$.

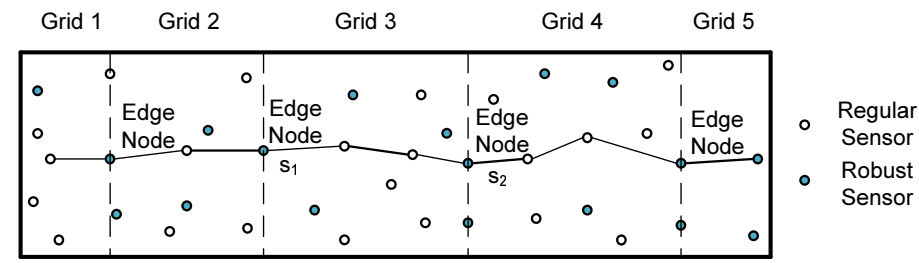

Fig. 4. Grid Division

Then robust sensor $s_{2}$ is selected as the coordinator for grid $a$.

After the selection, each coordinator checks the value of $\Delta n$ in the grid. If $\Delta n<0$, it means there are not enough mobile robust sensors to reconstruct the segment of barrier in the current grid. Then the coordinator merges two neighbor grids. After merging, a new coordinator is selected in the new grid using same method. Then the new coordinator checks the value of $\Delta n$ again. If the value of $\Delta n$ is still negative, the merging continues. Otherwise, it stops. One example is shown in Fig. 5. In the example, $s_{2}$ is the coordinator of grid $a$ as shown in Fig. 5(a). Since $\Delta n_{a}=-1<0, s_{2}$ merges the grid $a$ and $c$ to generate a new grid $a^{\prime}$ as shown in Fig. 5(b). In the new grid $a^{\prime}, s_{1}$ and $s_{3}$ are new edge nodes. After calculations, $s_{3}$ is selected as the coordinator of grid $a^{\prime}$ as shown in Fig. 5(c). Since $\Delta n_{a^{\prime}}=1>0$, grid merging stops. At the end of the algorithm, the entire area are divided into several grids, each of which has a coordinator, and contains enough mobile robust sensors to reconstruct the segment of barrier.

2) Virtual Movement Evaluation: When a grid contains enough mobile robust sensors, the next step is to choose appropriate robust sensors to reconstruct the segment of barrier. The reconstruction considering two strategies: repair the segment of barrier and reconstruct a new segment of barrier.

In the first strategy, mobile robust sensors are virtually moved to the positions that regular sensors used to stay in the barrier to repair the segment of barrier. The coordinator virtually moves the mobile robust sensors that are closest to the positions and records the energy consumption of the virtual movement. For example, as shown in Fig. 6(a), there are 3 regular sensors in the segment of barrier in grid $a$. Robust sensors $s_{1}, s_{2}$ and $s_{3}$ are selected to virtually move to the regular sensors' positions $p 1, p 2$ and $p 3$, respectively, as shown in Fig. 6(b), since they are the closest robust sensors to the 


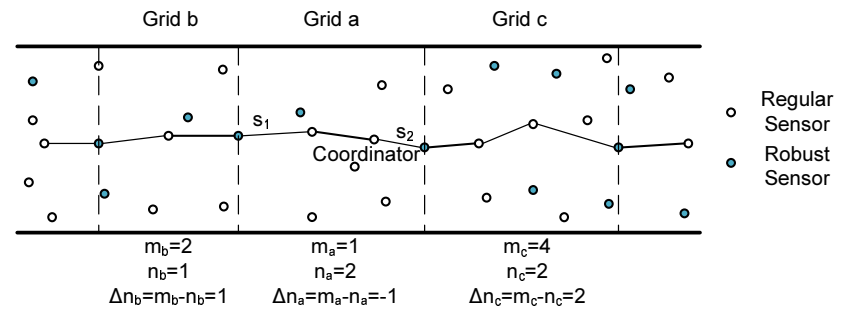

(a) Coordinator Selection

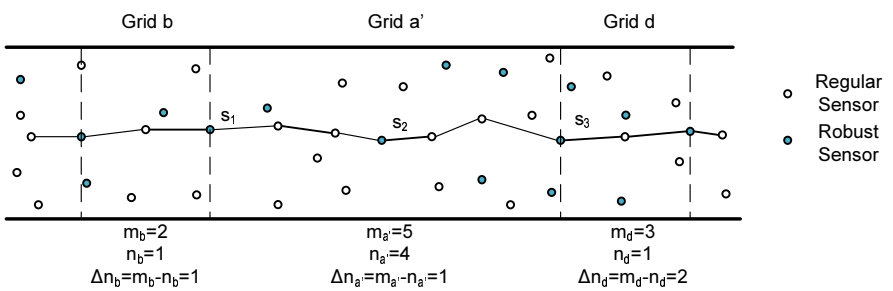

(b) Neighbor Grids Merging

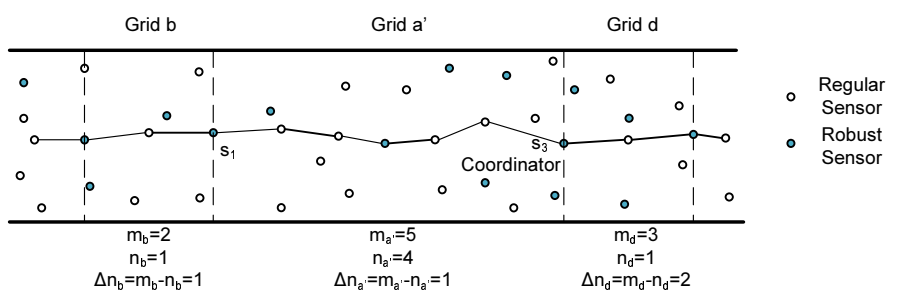

(c) Coordinator Reselection

Fig. 5. Grid Merging

positions. Fig. 6(c) is the repaired barrier by robust sensors in grid $a$.

In the second strategy, mobile robust sensors are virtually moved to certain positions to construct a new segment of barrier considering the shortest distance between two edge nodes in the grid. The coordinator first constructs a straight path between itself and the other edge node. It calculates the least number of mobile robust sensors to cover the path considering sensing overlaps are minimized, which is $\left\ulcorner\frac{L_{p}-2 R S}{2 R S}\right\urcorner$, where $L_{p}$ is the length of the path. The coordinator sets $\left\ulcorner\frac{L_{p}-2 R S}{2 R S}\right\urcorner$ positions starting from itself with the distance of $2 R S$ along the path. Then the coordinator virtually moves closest robust sensors to the positions and records the energy consumption. One example is shown in Fig. 7. The coordinator sets two positions $p_{1}^{\prime}$ and $p_{2}^{\prime}$ on the path and then virtually moves robust sensors $s_{1}$ and $s_{2}$ to them respectively as shown in Fig. 7(a). The barrier constructed by the second strategy is shown in Fig. 7(b).

After the coordinator simulates the movement and obtains energy consumption of both strategies, it picks up the strategy with the minimum energy consumption and notifies robust sensors to move to the calculated positions according to the strategy.

\section{Barrier Reconstruction Algorithm}

When sunny weather comes again, most regular sensors are survived after drying and come back to work. A new default barrier needs to be constructed by using both regular sensors

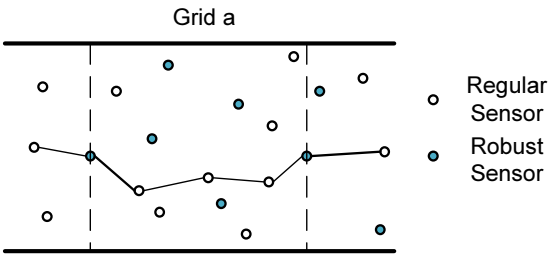

(a) Original Segment

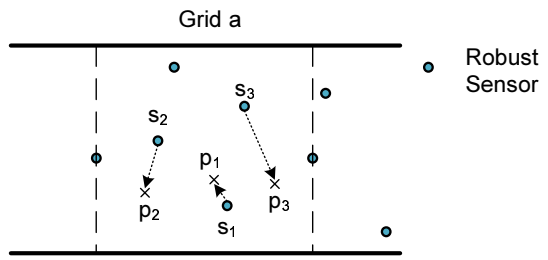

(b) First Strategy Movement

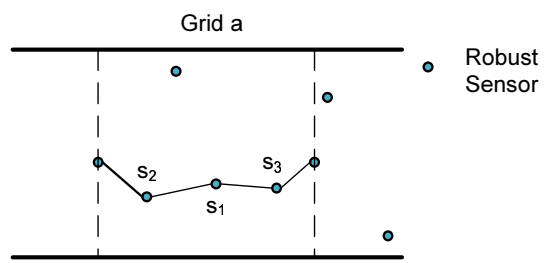

(c) First Strategy Finished

Fig. 6. Virtual Movement of First Strategy

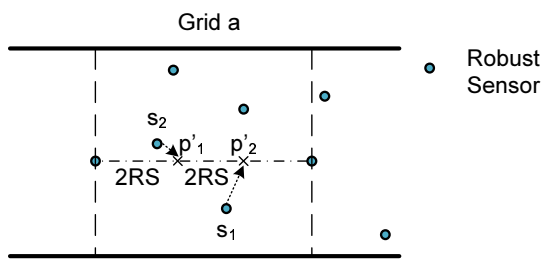

(a) Second Strategy Movement

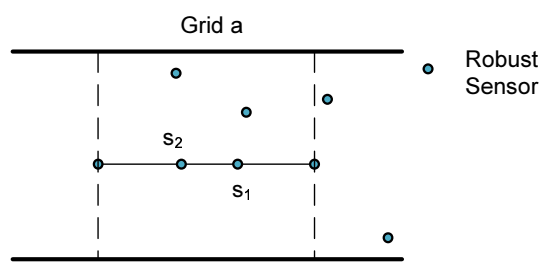

(b) Second Strategy Finished

Fig. 7. Virtual Movement of Second Strategy

and robust sensors. Otherwise, robust sensors will be overused. In each grid, the coordinator examines the regular sensors' survival status and determines whether a global construction is needed.

The coordinator first checks whether all the regular sensors in the old default barrier are survived after the rain. The old default barrier is the barrier working under the most recent sunny weather. If they are, they are scheduled to work again to form a new default barrier for the next sunny periods. Otherwise, if there is one regular sensor dead, the coordinator informs other coordinators to construct a new default barrier by using global information. The construction algorithm is 
the same as Default Barrier Construction Algorithm, which considers the least movement of robust sensors. Then the new default barrier is scheduled to work under the coming sunny period.

\section{Evaluation}

In this section, we evaluate the proposed greedy algorithm. Our objective is three-fold: (1) Evaluating our algorithm in minimizing number of moved robust sensors and moving distance of robust sensors under different weather conditions; (2) Evaluating our algorithm in minimizing total energy consumption of barrier construction under different weather conditions; (3) Evaluating our algorithm in prolonging network lifetime under different weather conditions.

To evaluate our algorithm, four metrics are employed: (1) total number of moved robust sensors, (2) total moving distance of robust sensors, (3) total energy consumption in barrier construction, and (4) network lifetime. The network lifetime is defined as the time that one barrier cannot be established.

We compare our algorithm with Mobile Barrier algorithm in [13] and Density Barrier algorithm in [14], which are the closet works to ours. MB algorithm uses both static sensors and mobile sensors to construct a barrier in the network. To save the energy of movement of mobile sensors, a barrier line is pre-defined. MB algorithm first constructs a barrier only using static sensors along the pre-defined barrier line. When it cannot find a static sensor to construct the barrier, a mobile sensor is moved to a certain position on the pre-defined line to continue the barrier construction considering least energy consumption in movement. In the evaluation, the pre-defined line in MB algorithm is a horizontal middle line in the field. DB algorithm first constructs a virtual barrier line using regression method based on the sensors' positions. Then a number of points are calculated on the line. After that, the mobile sensors move to the closest points on the virtual barrier to save energy. In the following, we will call them MB algorithm and $\mathrm{DB}$ algorithm for short, respectively. To be fair, both $\mathrm{MB}$ algorithm and $\mathrm{DB}$ algorithm know the weather forecast. They use both static regular sensors and mobile robust sensors to build a barrier under sunny weather, and only use mobile robust sensors to construct a barrier under rainy weather.

We evaluate the performance of the proposed algorithm through simulation. In the simulation, the long-belt shaped area is $1000 \mathrm{~m} \times 100 \mathrm{~m}$. Both regular sensors and robust sensors are randomly deployed in the area. By default, the number of regular sensor nodes $n_{g}$ and the number of robust sensors nodes $n_{r}$ are both set to 500 unless we study the impact of the number of sensors. For each sensor, its sensing range is $10 \mathrm{~m}$, and its communication range is $20 \mathrm{~m}$. The time span is divided into slots, each of which is 6 hours. By default, the network runs for 2 time slots unless we evaluate the network lifetime. The first slot is sunny weather and the second slot is rainy weather. Failure probability of regular sensors under rainy weather is set to $5 \%$. All simulation results are the average of 100 times. The network topology is randomly regenerated in each simulation.

\section{A. Evaluation of Number of Moved Robust Sensors}

We evaluate the total number of robust sensors that need to move in the barrier construction under different $n_{g}$ and $n_{r}$. First, $n_{r}$ varies from 500 to 2500 . The ratio of the number of robust sensors to the number of regular sensors is from $1: 1$ to $5: 1$. Fig. 8 shows the result. From the result, our algorithm always outperforms MB algorithm and DB algorithm. For example, when the ratio is $5: 1$, the number of moved robust sensors under our algorithm is only 13 , which is about $72 \%$ less than that under MB algorithm, which is 50 , and that under DB algorithm, which is 52 . It can also be seen that the number of moved robust sensors is always 50 under MB algorithm, and 52 under DB algorithm. In addition, the number of moved robust sensors under our algorithm decreases as $n_{r}$ increases. The reason is that the barrier line is pre-defined in MB algorithm, and thus there are always a fixed number of robust sensors moving to the pre-defined position under the rainy weather, no matter how dense they are. In DB algorithm, there is a very low probability that mobile sensors staying exactly on the positions of the virtual barrier line and thus the number of robust sensors moving to the pre-defined position is a fixed value. In our algorithm, as the number of robust sensors increases, the size of the grids becomes small and thus the number of robust sensors to be moved becomes small.

Second, $n_{g}$ varies from 500 to 5000 , which means the ratio of robust sensors to regular sensors is from $1: 1$ to $1: 10$. The result is shown in Fig. 9. From the result, our algorithm still outperforms MB algorithm and DB algorithm. For example, when the ratio is $1: 1$, the number of moved robust sensors under our algorithm is 36 , which is about $28 \%$ less than that under MB algorithm, which is 50 , and that under $\mathrm{DB}$ algorithm, which is 52 . It can also be observed that as $n_{g}$ increases, the number of moved sensors in our algorithm first increases and then keeps almost constant. The reason is that as the number of regular sensors increases, the number of regular sensors used to construct the barrier under the sunny weather increases in our algorithm and then the size of grids increases. As the size of grids becomes larger, the number of moved robust sensors increases. When the distribution of regular sensor reaches a certain high density, there is always a probability to incorporate a small number of robust sensors to construct the default barrier under the sunny weather. Thus the maximum number of robust sensors to be moved is almost constant.

\section{B. Evaluation of Moving Distance of Mobile Robust Sensors}

We evaluate the total moving distance of robust sensors under different $n_{g}$ and $n_{r}$. First, $n_{r}$ varies from 500 to 2500 . From the result in Fig. 10, the total moving distance under our algorithm is shorter than that under MB algorithm and that under DB algorithm. For example, when the ratio is $5: 1$, the total moving distance under our algorithm is $38 \mathrm{~m}$, which is about $76 \%$ less than that under MB algorithm, which is $159 \mathrm{~m}$, 


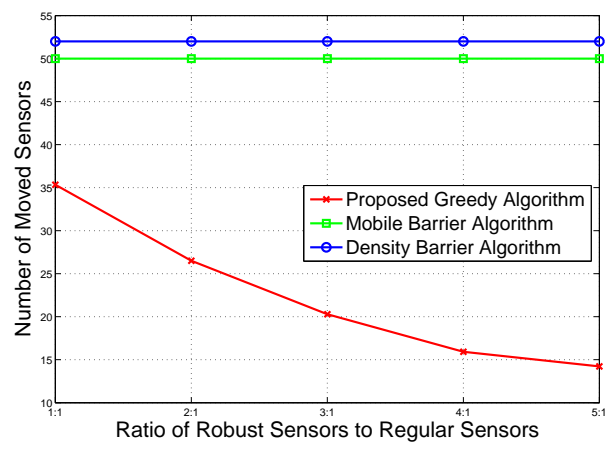

Fig. 8. Number of Moved Robust Sensor under different $n_{r}$

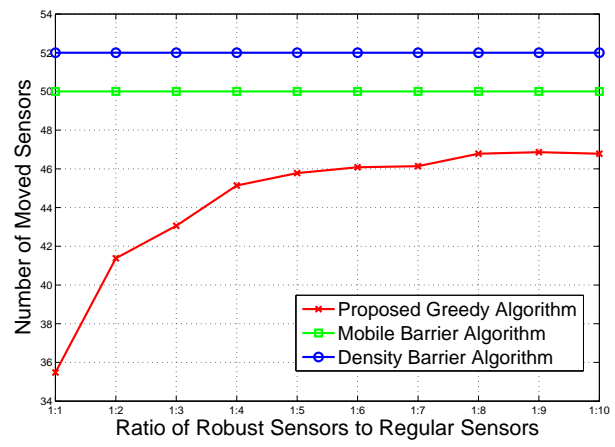

Fig. 9. Number of Moved Robust Sensor under different $n_{g}$

and that under DB algorithm, which is $173 \mathrm{~m}$. The reason is that as robust sensors become more dense, more robust sensors close to default barrier can be used to construct a new barrier under rainy weather in our algorithm. It is same for $\mathrm{MB}$ algorithm and DB algorithm. Thus the energy consumption decreases in both algorithms.

Second, $n_{g}$ varies from 500 to 5000 . The result is shown in Fig. 11. Still, the moving distance under our algorithm is shorter than that under both MB algorithm and DB algorithm. For example, when the ratio is $1: 1$, the total moving distance under our algorithm is $225 \mathrm{~m}$, which is about $38 \%$ less than that under MB algorithm, which is $360 \mathrm{~m}$, and $44 \%$ less than that under DB algorithm, which is $400 \mathrm{~m}$. From the result, it can be observed that as $n_{g}$ increases, the total moving distance keeps a constant value under both $\mathrm{MB}$ algorithm and DB algorithm. It first increases and then reaches a threshold under our algorithm. The reason is that under the random distribution, the average moving distance for each mobile robust sensor is almost a constant value. Thus the result of total moving distance has the same trend as the result of the number of moved robust sensors for all three algorithms.

\section{Evaluation of Total Energy Consumption}

We evaluate the total energy consumption in barrier construction under different $n_{g}$ and $n_{r}$. According to [33], $P_{s}$ is set to $0.5 J$, which is the energy consumption of starting the motor and $P_{e}$ is set to be $1.75 J$, which is the energy

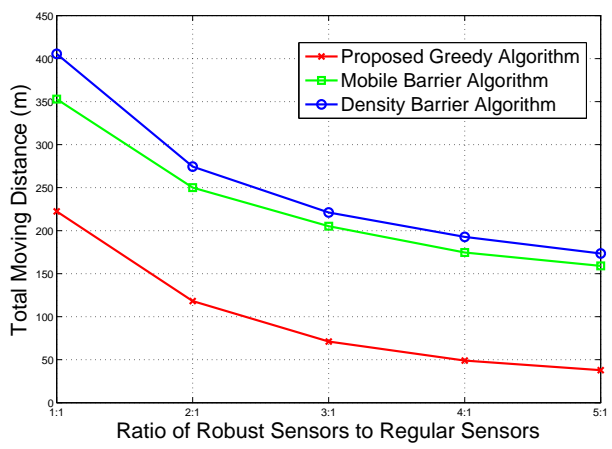

Fig. 10. Total moving distance under different $n_{r}$

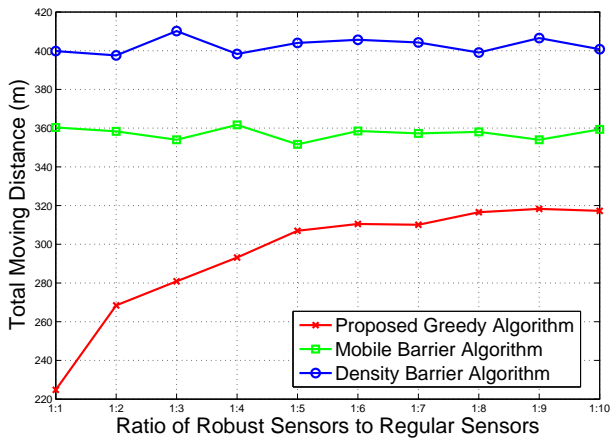

Fig. 11. Total moving distance under different $n_{g}$

consumption per meter movement. First, $n_{r}$ ranges from 500 to 2500 . Fig. 12 shows the result. Our algorithm consumes less energy than $\mathrm{MB}$ algorithm and $\mathrm{DB}$ algorithm. For example, when the ratio is $5: 1$, the energy consumption under our algorithm is $133 \mathrm{~J}$, which is about $75 \%$ less than that under MB algorithm, which is $537 \mathrm{~J}$, and about $81 \%$ less than that under DB algorithm, which is $712 \mathrm{~J}$. It can be also observed that as $n_{r}$ increases, the energy consumption under all three algorithms decreases. It is because the total moving distance decreases, which is shown previously.

Second, we evaluate the energy consumption under different densities of regular sensors. $n_{g}$ varies from 500 to 5000 . The result is shown in Fig. 13. Our algorithm still consumes less energy than both $\mathrm{MB}$ algorithm and $\mathrm{DB}$ algorithm. For example, when the ratio is $1: 1$, the energy consumption under our algorithm is $689 \mathrm{~J}$, which is about $37 \%$ less than that under MB algorithm, which is $1091 \mathrm{~J}$ and about $43 \%$ less than that under DB algorithm, which is $1203 \mathrm{~J}$. It can be observed that as $n_{g}$ increases, the energy consumption under all three algorithms reaches a threshold. The reason is that the total moving distance reaches a threshold, which is shown previously.

\section{Evaluation of Network Lifetime}

We evaluate the network lifetime under different $n_{g}$ and $n_{r}$. The weather conditions in the network are one sunny slot, one rainy slot, one sunny slot, one rainy slot, and so on so 


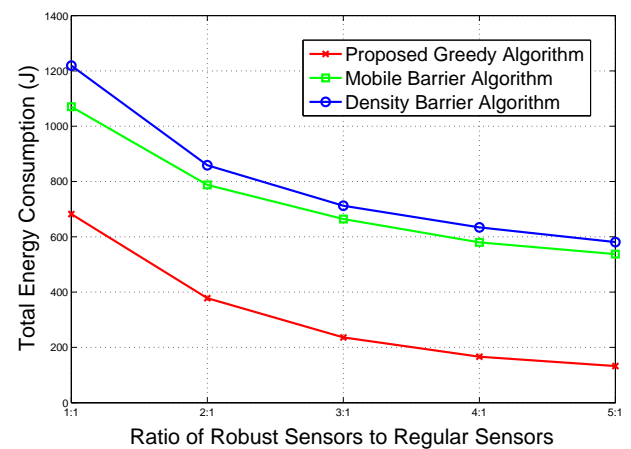

Fig. 12. Total Energy Consumption under different $n_{r}$

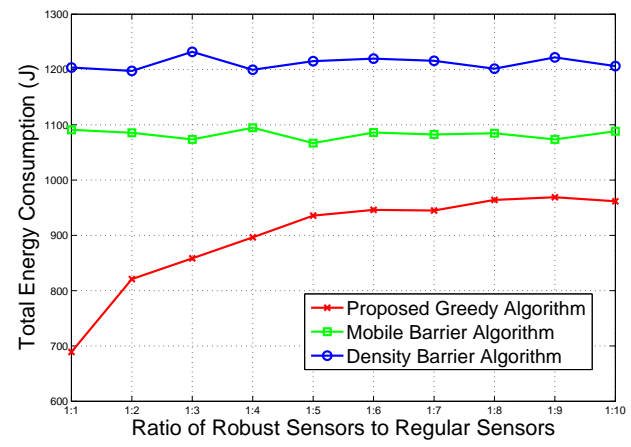

Fig. 13. Total Energy Consumption under different $n_{g}$

forth. First, $n_{r}$ varies from 500 to 2500 . The result is shown in Fig. 14. From the result, our algorithm achieves a longer network lifetime than $\mathrm{MB}$ algorithm and $\mathrm{DB}$ algorithm. For example, when the ratio is $5: 1$, the network lifetime is 424 hours under our algorithm, which is about $22 \%$ longer than that under MB algorithm, which is 349 hours, and about $112 \%$ longer than that under DB algorithm, which is 200 hours. It can be observed that the lifetime increases as $n_{r}$ increases. The reason is that as $n_{r}$ increases, the number of barriers can work under both rainy weather and sunny weather increases, and thus the network lifetime increases.

Second, $n_{g}$ varies from 500 to 5000 . From the result shown in Fig. 15, the network lifetime under our algorithm is still longer than that under $\mathrm{MB}$ algorithm and that under $\mathrm{DB}$ algorithm, since our algorithm can utilize regular sensors more effectively than MB algorithm and DB algorithm. For example, when the ratio is $1: 10$, the network lifetime is 117 hours under our algorithm, which is about $55 \%$ longer than that under MB algorithm, which is 76 hours, and $30 \%$ longer than that under DB algorithm, which is 90 hours. It can be seen that the lifetime increases as $n_{g}$ increases under both algorithms and reaches a threshold. It is because the energy consumption in barrier construction reaches a threshold, which is shown before.

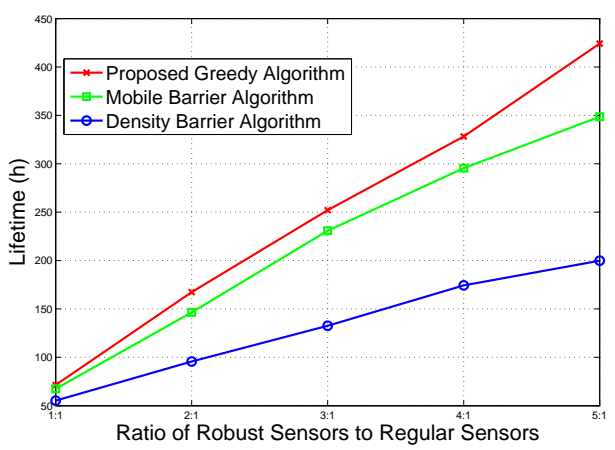

Fig. 14. Network Lifetime under different $n_{r}$

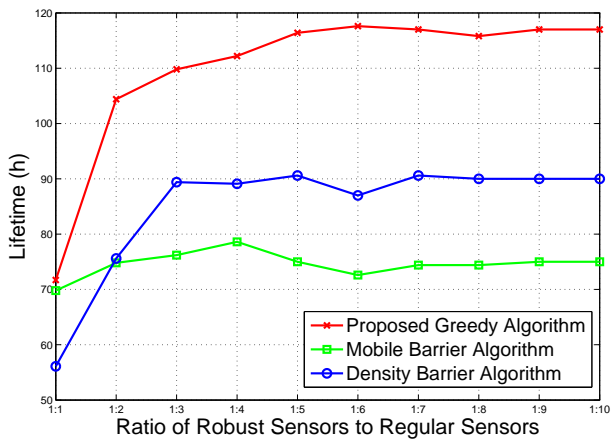

Fig. 15. Network Lifetime under different $n_{g}$

\section{CONCLUSION}

In this paper, we study the barrier coverage problem in a mobile survivability-heterogeneous wireless sensor network. A new greedy algorithm is proposed to maintain barrier coverage under both rainy and sunny weather in a long-belt shaped field. By adopting the weather forecast, the algorithm minimizes the energy consumption in the barrier construction under different weather conditions and thus prolongs network lifetime. The simulation results show that our algorithm outperforms the existing solutions.

\section{ACKNOWLEDGMENTS}

This work is supported by the National Science Foundation grants NSF-1128369.

\section{REFERENCES}

[1] M. Cardei, M. T. Thai, Y. Li, and W. Wu, "Energy-efficient target coverage in wireless sensor networks," in INFOCOM 2005. 24th Annual Joint Conference of the IEEE Computer and Communications Societies. Proceedings IEEE, vol. 3, 2005, pp. 1976-1984.

[2] H. Liu, P. Wan, C.-W. Yi, X. Jia, S. Makki, and N. Pissinou, "Maximal lifetime scheduling in sensor surveillance networks," in INFOCOM 2005. 24th Annual Joint Conference of the IEEE Computer and Communications Societies. Proceedings IEEE, vol. 4, 2005, pp. 2482-2491.

[3] J. Tian, T. Yan, X. Gao, and G. Wang, "Scheduling survivabilityheterogeneous sensor networks for critical location surveillance," $A C M$ Transactions on Sensor Networks, accepted to appear, 2015.

[4] L. Richardson, Weather Prediction by Numerical Process. Cambridge University Press, 2007. 
[5] K. Martinez, J. Hart, and R. Ong, "Environmental sensor networks," Computer, 2004.

[6] L. Selavo, A. Wood, Q. Cao, T. Sookoor, H. Liu, A. Srinivasan, Y. Wu, W. Kang, J. Stankovic, D. Young, and J. Porter, "Luster: Wireless sensor network for environmental research," in Proceedings of the 5th International Conference on Embedded Networked Sensor Systems, 2007, pp. 103-116.

[7] J. Tian, T. Yan, and G. Wang, "A network coding based energy efficient data backup in survivability-heterogeneous sensor networks," IEEE Transactions on Mobile Computing, available online, 2014

[8] Y. Wang, X. Wang, B. Xie, D. Wang, and D. P. Agrawal, "Intrusion detection in homogeneous and heterogeneous wireless sensor networks," IEEE Transactions on Mobile Computing, vol. 7, pp. 698-711, 2008.

[9] X. Wang, X. Wang, and J. Zhao, "Impact of mobility and heterogeneity on coverage and energy consumption in wireless sensor networks," in Distributed Computing Systems (ICDCS), 2011 31st International Conference on, 2011, pp. 477-487.

[10] M. Cardei, S. Yang, and J. Wu, "Algorithms for fault-tolerant topology in heterogeneous wireless sensor networks," IEEE Transactions on Parallel and Distributed Systems, vol. 19, pp. 545-558, 2008.

[11] X. Du and F. Lin, "Maintaining differentiated coverage in heterogeneous sensor networks," EURASIP Journal on Wireless Communications and Networking, no. 4, 2005.

[12] K. Shih, H. Chen, C. Chou, and B. Liu, "On target coverage in wireless heterogeneous sensor networks with multiple sensing units," Journal of Network and Computer Applications, vol. 32, no. 4, pp. 866-877, 2009.

[13] A. Saipulla, B. Liu, G. Xing, X. Fu, and J. Wang, "Barrier coverage with sensors of limited mobility," in MobiHoc, 2010, pp. 201-210.

[14] C.-F. Cheng, T.-Y. Wu, and H.-C. Liao, "A density-barrier construction algorithm with minimum total movement in mobile wsns," Computer Networks, vol. 62, pp. 208-220, 2014.

[15] S. Kumar, T. H. Lai, and A. Arora, "Barrier coverage with wireless sensors," in ACM MobiCom, 2005, pp. 284-298.

[16] A. Chen, S. Kumar, and T. H. Lai, "Designing localized algorithms for barrier coverage," in ACM MobiCom, 2007, pp. 63-74.

[17] B. Liu, O. Dousse, J. Wang, and A. Saipulla, "Strong barrier coverage of wireless sensor networks," in MobiHoc, 2008, pp. 411-420.

[18] A. Saipulla, C. Westphal, B. Liu, and J. Wang, "Barrier coverage of linebased deployed wireless sensor networks," in IEEE INFOCOM, 2009, pp. $127-135$.

[19] S. Kumar, T. H. Lai, M. E. Posner, and P. Sinha, "Maximizing the lifetime of a barrier of wireless sensors," IEEE Transactions on Mobile Computing, vol. 9, no. 8, pp. 1161-1172, 2010.

[20] A. Chen, S. Kumar, and T. H. Lai, "Local barrier coverage in wireless sensor networks," IEEE Transactions on Mobile Computing, vol. 9, no. 4, pp. 491-504, 2010.

[21] A. Chen, Z. Li, T. H. Lai, and C. Liu, "One-way barrier coverage with wireless sensors," in IEEE INFOCOM, 2011, pp. 626-630.

[22] J. Tian, W. Zhang, G. Wang, and X. Gao, "2d k-barrier duty-cycle scheduling for intruder detection in wireless sensor networks," Computer Communications, vol. 43, no. 0, pp. 31 - 42, 2014.

[23] G. Yang and D. Qiao, "Multi-round sensor deployment for guaranteed barrier coverage," in IEEE INFOCOM, 2010.

[24] S. Paul and N. K. Sao, "An energy efficient hybrid node scheduling scheme in cluster based wireless sensor networks," in Proceedings of the World Congress on Engineering, vol. 2, 2011.

[25] Y. Keung, B. Li, and Q. Zhang, "The intrusion detection in mobile sensor network," in MobiHoc, 2010, pp. 11-20.

[26] J. Tian, G. Wang, T. Yan, and W. Zhang, "Detect smart intruders in sensor networks by creating network dynamics," Computer Networks, vol. 62, no. 0, pp. $182-196,2014$.

[27] H. Shibo, C. Jiming, L. Xu, S. Xuemin, and S. Youxian, "Cost-effective barrier coverage by mobile sensor networks," in IEEE INFOCOM, 2012 , pp. 819-827.

[28] Vaisala, "User's guide vaisala drycap dewpoint transmitter dmt143," 2015. [Online]. Available: www.vaisala.com

[29] C. Tao, Y. Zheng, L. Yunhao, G. Deke, and L. Xueshan, "Localization in non-localizable sensor and ad-hoc networks: A localizability-aided approach," in INFOCOM, 2011 Proceedings IEEE, 2011, pp. 276-280.

[30] W. Liu, D. Wang, H. Jiang, W. Liu, and C. Wang, "Approximate convex decomposition based localization in wireless sensor networks," in INFOCOM, 2012 Proceedings IEEE, 2012, pp. 1853-1861.

[31] M. Huang, S. Chen, and Y. Wang, "Minimum cost localization problem in wireless sensor networks," in IEEE SECON, 2010, pp. 1-9.
[32] X. Hu, Y. Hu, and B. Xu, "Energy-balanced scheduling for target tracking in wireless sensor networks," ACM Transaction on Sensor Networks (TOSN), vol. 11, no. 1, pp. 21:1-21:29, 2014.

[33] G. Wang, M. J. Irwin, P. Berman, H. Fu, and T. La Porta, "Optimizing sensor movement planning for energy efficiency," in Low Power Electronics and Design, 2005. ISLPED'05. Proceedings of the 2005 International Symposium on, 2005, pp. 215-220.

[34] A. Lorenc, "Analysis methods for numerical weather prediction," Quarterly Journal of the Royal Meteorological Society, 1986. 
Jie Tian received the B.S. degree in Computer Science from Tianjin University, Tianjin, China, in 2005, and got his M.S. degree in Computer Science at Nankai University, Tianjin, China, in 2008. He is currently a Ph.D. candidate in Department of Computer Science at New Jersey Institute of Technology. His research includes wireless networks, ad hoc/sensor network and mobile computing.

Xiaoyuan Liang is currently a Ph.D. student at Computer Science Department in New Jersey Institute of Technology since 2013. He received his bachelor degree from Harbin Institute of Technology in China.

Guiling Wang received the B.S. degree in software from Nankai University, Tianjin, China, and the Ph.D. degree in computer science and engineering with a minor in statistics from The Pennsylvania State University, State College, PA, USA, in 2006. She joined the New Jersey Institute of Technology, Newark, NJ, USA, in the fall of 2006 and was promoted to Associate Professor with tenure in June 2011. 


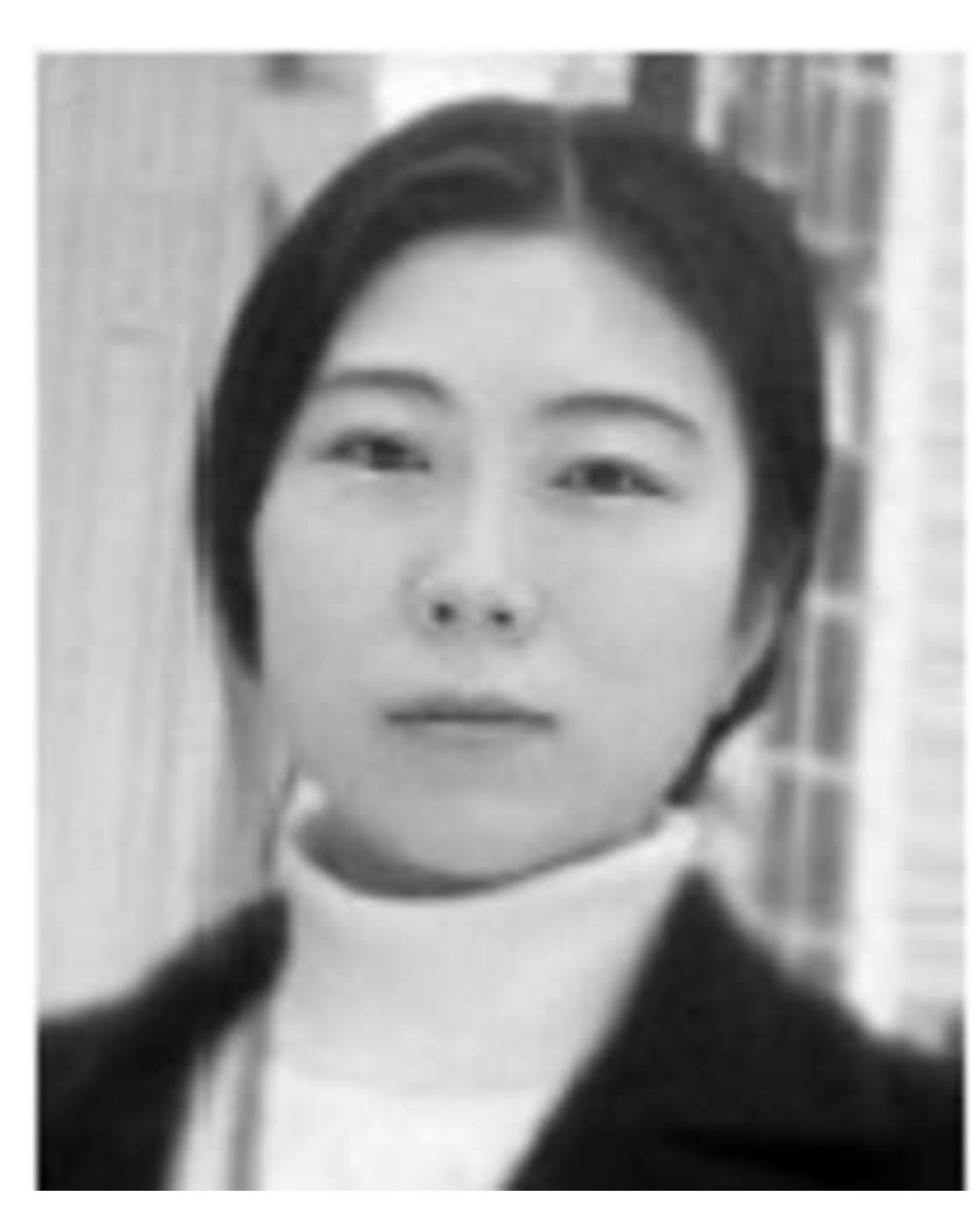 \\ guiling_wang.jpg}
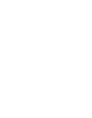


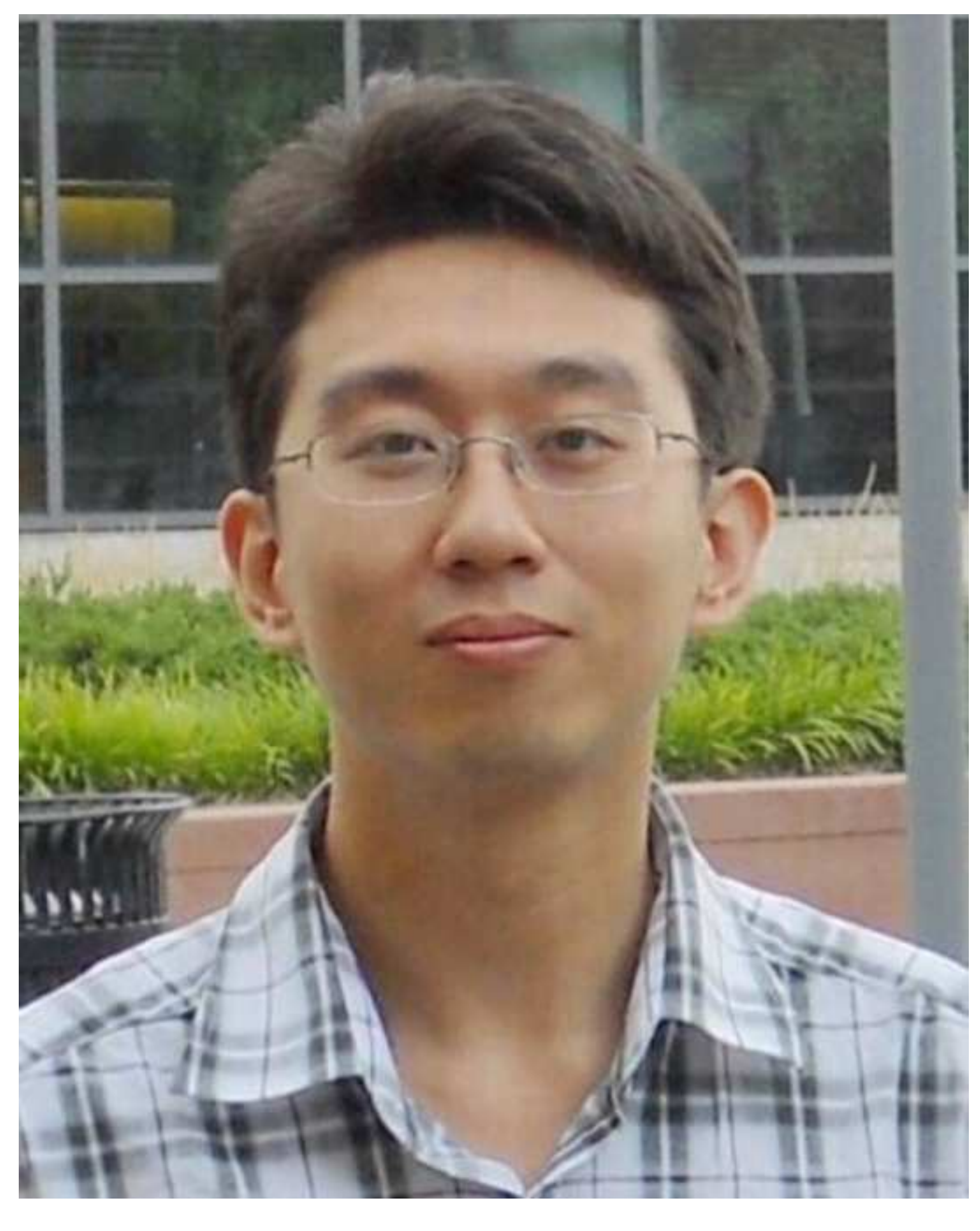

\section{jle_tian.jpg}

g
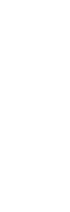


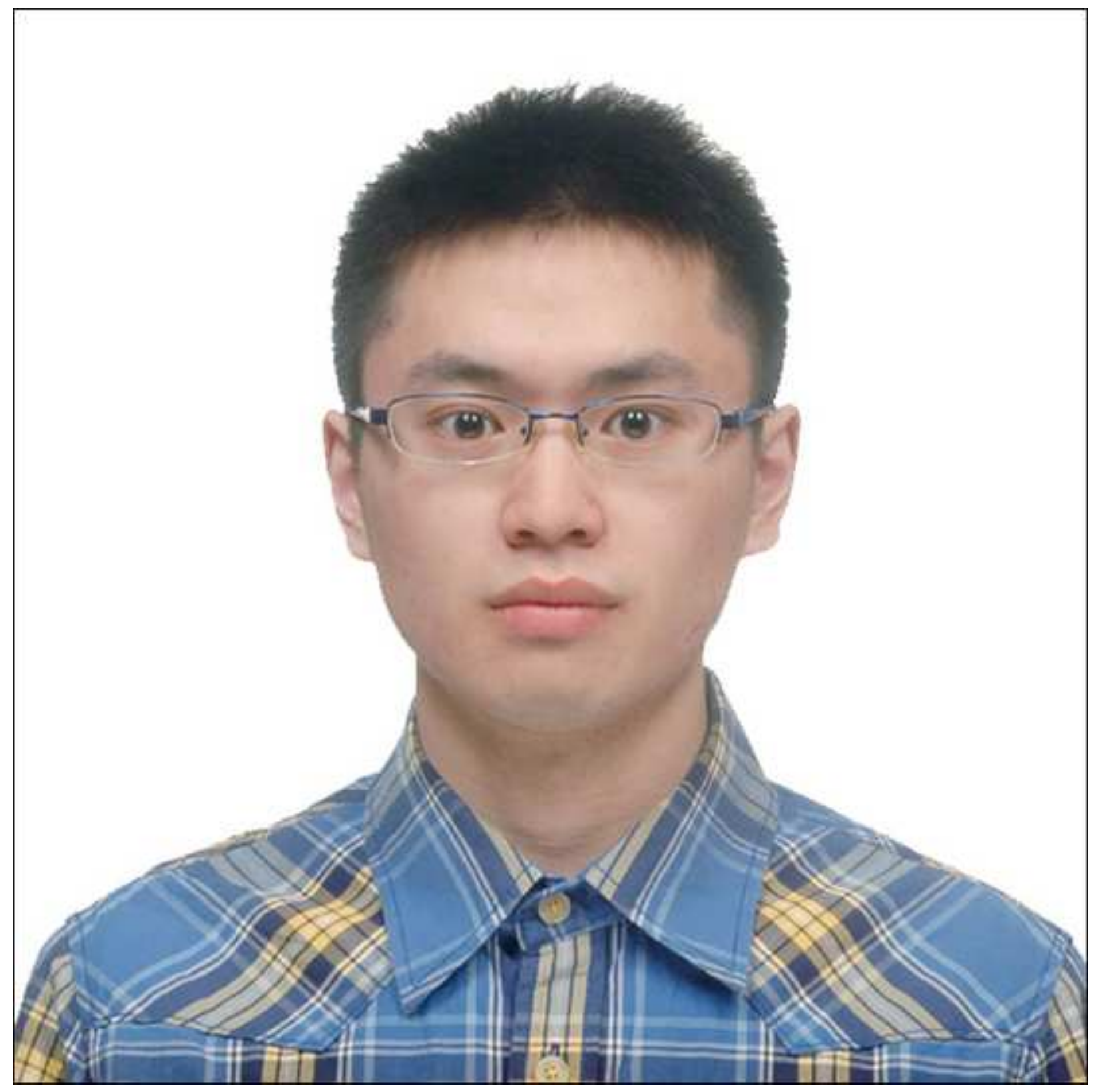

